\title{
Berezin Quantization and Holomorphic Representations
}

\author{
Benjamin Cahen (*)
}

ABstract - Let $G$ be a quasi-Hermitian Lie group and let $\pi$ be a unitary highest weight representation of $G$ realized in a reproducing kernel Hilbert space of holomorphic functions. We study the Berezin symbol map $S$ and the corresponding Stratonovich-Weyl map $W$ which is defined on the space of HilbertSchmidt operators acting on the space of $\pi$, generalizing some results that we have already obtained for the holomorphic discrete series representations of a semi-simple Lie group. In particular, we give explicit formulas for the Berezin symbols of the representation operators $\pi(g)$ (for $g \in G$ ) and $d \pi(X)$ (for $X$ in the Lie algebra of $G$ ) and we show that $S$ provides an adapted Weyl correspondence in the sense of [B. Cahen, Weyl quantization for semidirect products, Differential Geom. Appl. 25 (2007), 177-190]. Moreover, in the case when $G$ is reductive, we prove that $W$ can be extended to the operators $d \pi(X)$ and we give the expression of $W(d \pi(X))$. As an example, we study the case when $\pi$ is a generic unitary representation of the diamond group.

Mathematics Subject Classification (2010). 22E46; 32M10; 81S10; 46E22; $32 \mathrm{M} 15$.

KEYworDs. Berezin quantization; Berezin transform; quasi-Hermitian Lie group; coadjoint orbit; unitary representation; holomorphic representation; reproducing kernel Hilbert space; coherent states; Weyl correspondence; StratonovichWeyl correspondence.

\section{Introduction}

In [11] and [12], we introduced the notion of adapted Weyl correspondence in order to generalize the usual Weyl quantization [1], [28]. Let us consider a connected Lie group $G$ with Lie algebra $g$ and a unitary irre-

(*) Indirizzo dell'A.: Université de Metz, UFR-MIM, Département de mathématiques, LMMAS, ISGMP-Bât. A, Ile du Saulcy 57045, Metz cedex 01, France.

E-mail: cahen@univ-metz.fr 
ducible representation of $G$ on a Hilbert space $\mathcal{H}$. Assume that $\pi$ is associated with a coadjoint orbit $\mathcal{O} \subset \mathrm{g}^{*}$ of $G$ by the Kirillov-Kostant method of orbits [34], [35]. In [13], we gave the following definition for the notion of adapted Weyl correspondence (see also [31]).

DEFINITION 1.1. An adapted Weyl correspondence is an isomorphism $W$ from a vector space $\mathcal{A}$ of complex-valued smooth functions on the orbit $\mathcal{O}$ (called symbols) onto a vector space $\mathcal{B}$ of (not necessarily bounded) linear operators on $\mathcal{H}$ satisfying the following properties:

(1) the elements of $\mathcal{B}$ preserve a fixed dense domain $\mathcal{D}$ of $\mathcal{H}$;

(2) the constant function 1 belongs to $\mathcal{A}$, the identity operator I belongs to $\mathcal{B}$ and $W(1)=I$;

(3) $A \in \mathcal{B}$ and $B \in \mathcal{B}$ implies $A B \in \mathcal{B}$;

(4) for each $f$ in $\mathcal{A}$ the complex conjugate $\bar{f}$ of $f$ belongs to $\mathcal{A}$ and the adjoint of $W(f)$ is an extension of $W(\bar{f})$;

(5) the elements of $\mathcal{D}$ are $C^{\infty}$-vectors for the representation $\pi$, the functions $\tilde{X}(X \in \mathfrak{g})$ defined on $\mathcal{O}$ by $\tilde{X}(\xi)=\langle\xi, X\rangle$ are in $\mathcal{A}$ and $W(i \tilde{X}) v=d \pi(X) v$ for each $X \in \mathfrak{g}$ and each $v \in \mathcal{D}$.

A typical example is the case when $G$ is a connected simply-connected nilpotent Lie group. Each coadjoint orbit $\mathcal{O}$ of $G$ is then diffeomorphic to $\mathbb{R}^{2 n}$ where $n=1 / 2 \operatorname{dim} \mathcal{O}$, the unitary irreducible representation of $G$ associated with $\mathcal{O}$ can be realized in $L^{2}\left(\mathbb{R}^{n}\right)$ and the usual Weyl correspondence provides an adapted Weyl correspondence on $\mathcal{O}$ [4], [44]. Another construction of adapted Weyl correspondences in the nilpotent case can be found in [40]. Let us also mention that in [6] a Weyl correspondence on a finite dimensional coadjoint orbit of some infinite dimensional Lie group was used to recover the magnetic Weyl calculus.

Besides the nilpotent case, adapted Weyl correspondences have been constructed in various situations, in particular for principal series representations [11], [14], and for unitary representations of semi-direct products of the form $V \rtimes K$ where $K$ is a semi-simple Lie group acting linearly on a vector space $V$ [13], [21], [22]. Note that adapted Weyl correspondences have different applications in harmonic analysis and deformation theory as the construction of covariant star-products on coadjoint orbits [11] and the study of contractions of Lie group unitary representations [25], [17].

Another way to generalize the usual quantization rules is the notion of Stratonovich-Weyl correspondence [42], [29]. J. M. Gracia-Bondìa, J. C. 
Vàrilly and various collaborators have studied systematically Stratonovich-Weyl correspondences [29], [24], [27], [30] (see also [10]).

DeFinition 1.2. [29], [30] Let $G$ be a Lie group and $\pi$ a unitary representation of $G$ on a Hilbert space $\mathcal{H}$. Let $M$ be a homogeneous $G$-space, for instance a coadjoint orbit $\mathcal{O}$ associated with $\pi$ as above, and let $\mu$ be a (suitably normalized) G-invariant measure on M. Then a StratonovichWeyl correspondence for the triple $(G, \pi, M)$ is an isomorphism $W$ from a vector space of operators on $\mathcal{H}$ to a space of (generalized) functions on $M$ satisfying the following properties:

(1) $W$ maps the identity operator of $\mathcal{H}$ to the constant function 1 ;

(2) the function $W\left(A^{*}\right)$ is the complex-conjugate of $W(A)$;

(3) Covariance: we have $W\left(\pi(g) A \pi(g)^{-1}\right)(x)=W(A)\left(g^{-1} \cdot x\right)$;

(4) Traciality: we have

$$
\int_{M} W(A)(x) W(B)(x) d \mu(x)=\operatorname{Tr}(A B) .
$$

In fact, each of these two notions of Weyl correspondence has advantages and disadvantages. For physical applications and for the Fourier theory of $G$, it is convenient to use a Stratonovich-Weyl correspondence instead of an adapted Weyl correspondence [27], [10]. On the other hand, adapted Weyl correspondences have the advantage to connect $\pi$ and $\mathcal{O}$ directly, that is suitable to study contractions.

When $G$ is a connected semi-simple non-compact real Lie group $G$ with finite center and $\pi$ is a holomorphic discrete series representation of $G$, we showed that the Berezin symbol calculus $S$ provides a $G$-equivariant adapted Weyl correspondence [18]. Moreover, $S$ is an isomorphism from the Hilbert space of all Hilbert-Schmidt operators on $\mathcal{H}$ (endowed with the Hilbert-Schmidt norm) onto a space of square-integrable functions on $\mathcal{O}$ and, in [20], we obtained a Stratonovich-Weyl correspondence $W$ by taking the isometric part in the polar decomposition of $S$, that is, $W:=\left(S S^{*}\right)^{-1 / 2} S$. Note that $B:=S S^{*}$ is the so-called Berezin transform which have been intensively studied, see for instance [26], [37], [38], [43], [45]. The idea of constructing Stratonovich-Weyl correspondences in such a way can be found in [27], see also [2] and [3]. In [20], we proved that $B$ hence $W$ can be extended to a class of functions which contains $S(d \pi(X))$ for $X \in \mathrm{g}^{c}$ and that the linear forms $X \rightarrow S(d \pi(X))$ and $X \rightarrow W(d \pi(X))$ are proportional. The same results also hold for unitary irreducible representations of a compact semi-simple Lie group, see [16] and [19]. 
The aim of the present paper is to extend the preceding results to the more general setting of [36], Chapter XII. More precisely, we consider a quasi-Hermitian Lie group $G$ and a unitary representation $\pi$ of $G$ which is realized in a reproducing kernel Hilbert space of holomorphic functions on some complex domain $\mathcal{D}$. We introduce and study the corresponding Berezin calculus $S$ and we compute the Berezin symbols of $\pi(g)$ for $g \in G$ and of $d \pi(X)$ for $X \in \mathfrak{g}$ (Section 4). We show that the coadjoint orbit $\mathcal{O}$ associated with $\pi$ is diffeomorphic to $\mathcal{D}$ and that $S$ provides an adapted Weyl correspondence on $\mathcal{O}$ (Section 5).

When $\mathrm{g}$ is reductive, we show that the corresponding Berezin transform $B$ can be extended to a space of functions which contains, in particular, $S(d \pi(X))$ for each $X \in \mathfrak{g}$. This allows us to define $W(d \pi(X))$ for $X \in \mathfrak{g}$. More precisely, consider the decomposition $\mathfrak{g}=\mathcal{Z}(\mathfrak{g}) \oplus \mathfrak{g}_{1} \oplus \ldots \oplus \mathfrak{g}_{n}$ where $\mathcal{Z}(\mathfrak{g})$ is the center of $g$ and the $\mathfrak{g}_{j}$ are simple ideals. Then we show that for each $j$ such that $g_{j}$ is non-compact there exists a constant $c_{j}>0$ such that $W(d \pi(X))=c_{j} S(d \pi(X))$ for each $X \in \mathfrak{g}_{j}$ (Section 6).

In the general case, it seems difficult to obtain an explicit expression for $W(d \pi(X))$. In Section 7 we illustrate the case where $g$ is solvable by taking $G$ to be the diamond group and $\pi$ to be a unitary irreducible representation of $G$ which is associated with a generic coadjoint orbit of $G$. In this case, the Berezin transform has a rather simple expression and we can compute $W(d \pi(X))$ for $X \in \mathfrak{g}$. Then we see in particular that $W(d \pi(X))$ and $S(d \pi(X))$ are not related in the same way as in the case where $g$ is reductive.

\section{Preliminaries}

The material of this section and of the following section is essentially taken from the excellent book of K.-H. Neeb, [36], Chapter VIII and Chapter XII (see also [41], Chapter II and, for the Hermitian case, [32], Chapter VIII and [33], Chapter 6).

Let $g$ be a real quasi-Hermitian Lie algebra, that is, a real Lie algebra for which the centralizer in $\mathfrak{g}$ of the center $\mathcal{Z}(\mathfrak{f})$ of a maximal compactly embedded subalgebra $\mathfrak{f}$ coincides with $\mathfrak{f}$ [36], p. 241. We assume that $\mathfrak{g}$ is not compact. Let $\mathfrak{g}^{c}$ be the complexification of $\mathfrak{g}$ and $Z=X+i Y \rightarrow Z^{*}=-X+i Y$ the corresponding involution. We fix a compactly embedded Cartan subalgebra $\mathfrak{h} \subset \mathfrak{f}$, [36], p. 241 and we denote by $\mathfrak{h}^{c}$ the corresponding Cartan subalgebra of $\mathrm{g}^{c}$. We write $\Delta:=\Delta\left(\mathfrak{g}^{c}, \mathfrak{h}^{c}\right)$ for the set of roots of $\mathfrak{g}^{c}$ relative to $\mathfrak{h}^{c}$ and $\mathfrak{g}^{c}=\mathfrak{h}^{c} \oplus \sum_{\alpha \in \Delta} \mathfrak{g}_{\alpha}$ 
for the root space decomposition of $\mathrm{g}^{c}$. Note that $\alpha(\mathfrak{h}) \subset i \mathbb{R}$ for each $\alpha \in \Delta$ [36], p. 233. Recall that a root $\alpha \in \Delta$ is called compact if $\alpha\left(\left[Z, Z^{*}\right]\right)>0$ holds for some element $Z \in \mathfrak{g}_{\alpha}$. All other roots are called non-compact [36], p. 235. We write $\Delta_{k}$, respectively $\Delta_{p}$, for the set of compact, respectively non-compact, roots. Note that $\mathfrak{f}^{c}=\mathfrak{h}^{c} \oplus \sum_{\alpha \in \Delta_{k}} \mathfrak{g}_{\alpha}$ [36], p. 235. Recall also that a subset $\Delta^{+} \subset \Delta$ is called a positive system if there exists an element $X_{0} \in i \mathfrak{h}$ such that $\Delta^{+}=\left\{\alpha \in \Delta: \alpha\left(X_{0}\right)>0\right\}$ and $\alpha\left(X_{0}\right) \neq 0$ for all $\alpha \in \Delta$. A positive system is then said to be adapted if for $\alpha \in \Delta_{k}$ and $\beta \in \Delta^{+} \cap \Delta_{p}$ we have $\beta\left(X_{0}\right)>\alpha\left(X_{0}\right)$, [36], p. 236. Here we fix a positive adapted system $\Delta^{+}$and we set $\Delta_{p}^{+}:=\Delta^{+} \cap \Delta_{p}$ and $\Delta_{k}^{+}:=\Delta^{+} \cap \Delta_{k}$, see [36], p. 241.

Let $G^{c}$ be a simply connected complex Lie group with Lie algebra $\mathrm{g}^{c}$ and $G \subset G^{c}$, respectively, $K \subset G^{c}$, the analytic subgroup corresponding to $\mathfrak{g}$, respectively, f́. We also set $K^{c}=\exp \left(\mathfrak{f}^{c}\right) \subset G^{c}$ as in [36], p. 506.

Let $\mathfrak{p}^{+}=\sum_{\alpha \in \Delta_{p}^{+}} \mathfrak{g}_{\alpha}$ and $\mathfrak{p}^{-}=\sum_{\alpha \in \Delta_{p}^{+}} \mathfrak{g}_{-\alpha}$. We denote by $P^{+}$and $P^{-}$the analytic subgroups of $G^{c}$ with Lie algebras $\mathfrak{p}^{+}$and $\mathfrak{p}^{-}$. Then $G$ is a group of the Harish-Chandra type [36], p. 507, that is, the following properties are satisfied:

(1) $\mathfrak{g}^{c}=\mathfrak{p}^{+} \oplus \mathfrak{f}^{c} \oplus \mathfrak{p}^{-}$is a direct sum of vector spaces, $\left(\mathfrak{p}^{+}\right)^{*}=\mathfrak{p}^{-}$and $\left[\mathfrak{f}^{+}, \mathfrak{p}^{ \pm}\right] \subset \mathfrak{p}^{ \pm}$

(2) The multiplication map $P^{+} K^{c} P^{-} \rightarrow G^{c},(z, k, y) \rightarrow z k y$ is a biholomorphic diffeomorphism onto its open image;

(3) $G \subset P^{+} K^{c} P^{-}$and $G \cap K^{c} P^{-}=K$.

Moreover, there exists an open connected $K$-invariant subset $\mathcal{D} \subset \mathfrak{p}^{+}$such that $G K^{c} P^{-}=\exp (\mathcal{D}) K^{c} P^{-}$, [36], p. 497. We denote by $\zeta: P^{+} K^{c} P^{-} \rightarrow P^{+}$, $\kappa: P^{+} K^{c} P^{-} \rightarrow K^{c}$ and $\eta: P^{+} K^{c} P^{-} \rightarrow P^{-}$the projections onto $P^{+}-, K^{c}$ and $P^{-}$-component. For $Z \in \mathfrak{p}^{+}$and $g \in G^{c}$ with $g \exp Z \in P^{+} K^{c} P^{-}$, we define the element $g \cdot Z$ of $\mathfrak{p}^{+}$by $g \cdot Z:=\log \zeta(g \exp Z)$. Note that we have $\mathcal{D}=G \cdot 0$.

We also denote by $g \rightarrow g^{*}$ the involutive anti-automorphism of $G^{c}$ which is obtained by exponentiating $X \rightarrow X^{*}$. We denote by $p_{\mathfrak{p}^{+}}, p_{\mathfrak{f}^{c}}$ and $p_{\mathfrak{p}^{-}}$the projections of $\mathfrak{g}^{c}$ onto $\mathfrak{p}^{+}, \mathfrak{f}^{c}$ and $\mathfrak{p}^{-}$associated with the direct decomposition $\mathfrak{g}^{c}=\mathfrak{p}^{+} \oplus \mathfrak{f}^{c} \oplus \mathfrak{p}^{-}$.

The $G$-invariant measure on $\mathcal{D}$ is $d \mu(Z):=\chi_{0}\left(\kappa\left(\exp Z^{*} \exp Z\right)\right) d \mu_{L}(Z)$ where $\chi_{0}$ is the character on $K^{c}$ defined by $\chi_{0}(k)=\operatorname{Det}_{\mathfrak{p}^{+}}(\operatorname{Ad} k)$ and $d \mu_{L}(Z)$ is a Lebesgue measure on $\mathcal{D}$ [36], p. 538 (in fact, this result is proved in [36] under the assumption that $\mathfrak{p}^{+}$is abelian but by adapting the arguments of [16] we see that the same result holds in the general case). 


\section{Representations}

We keep the notation of Section 2. We fix a unitary character $\chi$ of $K$. We also denote by $\chi$ the extension of $\chi$ to $K^{c}$. We set $K_{\chi}(Z, W)=$ $\chi\left(\kappa\left(\exp W^{*} \exp Z\right)\right)^{-1}$ for $Z, W \in \mathcal{D}$ and $J_{\chi}(g, Z)=\chi(\kappa(g \exp Z))$ for $g \in G$ and $Z \in \mathcal{D}$. We consider the Hilbert space $\mathcal{H}_{\chi}$ of holomorphic functions on $\mathcal{D}$ such that

$$
\|f\|_{\chi}^{2}:=\int_{\mathcal{D}}|f(Z)|^{2} K_{\chi}(Z, Z)^{-1} d \mu(Z)<+\infty
$$

In the rest of the paper, we assume that $\mathcal{H}_{\chi} \neq(0)$. Then $\mathcal{H}_{\chi}$ contains the polynomials [36], p. 546. Moreover, the formula

$$
\pi_{\chi}(g) f(Z)=J_{\chi}\left(g^{-1}, Z\right)^{-1} f\left(g^{-1} \cdot Z\right)
$$

defines a unitary representation of $G$ on $\mathcal{H}_{\chi}$ which is a highest weight representation with highest weight $\lambda:=\left.d \chi\right|_{\mathfrak{h}^{c}}$ [36], p. 540.

Note also that $\mathcal{H}_{\chi}$ is a reproducing kernel Hilbert space. More precisely, there exists a constant $c_{\chi}>0$ such that if we set $e_{Z}(W):=$ $c_{\chi} K_{\chi}(W, Z)$ then we have the reproducing property $f(Z)=\left\langle f, e_{Z}\right\rangle_{\chi}$ for each $f \in \mathcal{H}_{\chi}$ and each $Z \in \mathcal{D}$ [36], p. 540. Here $\langle\cdot, \cdot\rangle_{\chi}$ denotes the inner product on $\mathcal{H}_{\chi}$.

Applying the reproducing property to the constant function $f(Z)=1$ we see that $c_{\chi}$ is given by

$$
c_{\chi}^{-1}=\int_{\mathcal{D}} K_{\chi}(Z, Z)^{-1} d \mu(Z) .
$$

The following lemma will be need later.

Lemma 3.1. Let $E_{1}, E_{2}, \ldots, E_{m}$ be a basis of $\mathfrak{p}^{+}$such that $E_{j} \in \mathfrak{g}_{\alpha_{j}}$ where $\alpha_{j} \in A_{p}^{+}$for $j=1,2, \ldots, m$. Then we have $\lambda\left(\left[E_{j}^{*}, E_{j}\right]\right)<0$ for each $j=1,2, \ldots, m$.

Proof. For $Z \in \mathfrak{p}^{+}$, we write $Z=\sum_{j=1}^{m} z_{j} E_{j}$ and we set $p_{j}(Z)=z_{j}$. We also set $|Z|=\left(\sum_{j=1}^{m}\left|z_{j}\right|^{2}\right)^{1 / 2}$.

First, we fix $j=1,2, \ldots m$. We note that if $k=\exp H$ with $H \in \mathfrak{h}$ then we have $p_{j}(\operatorname{Ad}(k) Z)=e^{\alpha_{j}(H)} p_{j}(Z)$. Then, performing the change of variables 
$Z \rightarrow k \cdot Z=\operatorname{Ad}(k) Z$ in the integral

$$
\left\langle 1, p_{j}\right\rangle_{\chi}=\int_{\mathcal{D}} \overline{z_{j}} K_{\chi}(Z, Z)^{-1} d \mu(Z),
$$

we get $\left\langle 1, p_{j}\right\rangle_{\chi}=e^{-\alpha_{j}(H)}\left\langle 1, p_{j}\right\rangle_{\chi}$ for each $H \in \mathfrak{h}$ hence $\left\langle 1, p_{j}\right\rangle_{\chi}=0$.

Consequently, we can choose an orthonormal basis $\left(f_{p}\right)_{p \geq 0}$ of $\mathcal{H}_{\chi}$ such that $f_{0}(Z)=1 /\|1\|_{\chi}=c_{\chi}^{1 / 2}$ and $f_{1}(Z)=\left\|p_{j}\right\|^{-1} p_{j}(Z)$. It is well-known that the reproducing kernel of $\mathcal{H}_{\chi}$ is also given by $e_{Z}(W)=\sum_{l>0} \overline{f_{l}(Z)} f_{l}(W)$. Then we have

$$
e_{Z}(Z)=c_{\chi} \chi\left(\kappa\left(\exp Z^{*} \exp Z\right)\right)^{-1} \geq c_{\chi}+\left\|p_{j}\right\|^{-2}\left|z_{j}\right|^{2}
$$

for each $j=1,2, \ldots, m$.

On the other hand, by adapting the arguments of [15], Section 4, we get $\kappa\left(\exp Z^{*} \exp Z\right)=\exp \left(p_{\mathfrak{f}^{c}}\left(\left[Z^{*}, Z\right]\right)+O\left(|Z|^{3}\right)\right)$ for $Z$ close to 0 . Then we have

$$
c_{\chi} \chi\left(\kappa\left(\exp Z^{*} \exp Z\right)\right)^{-1}=c_{\chi}\left(1-p_{\mathrm{f}^{c}}\left(\left[Z^{*}, Z\right]\right)+O\left(|Z|^{3}\right)\right) .
$$

Thus, combining (3.1) and (3.2) and applying to $Z=z_{j} E_{j}$ for $z_{j}$ close to 0 , we obtain

$$
-\lambda\left(\left[E_{j}^{*}, E_{j}\right]\right)\left|z_{j}\right|^{2}+O\left(\left|z_{j}\right|^{3}\right) \geq c_{\chi}^{-1}\left\|p_{j}\right\|^{-2}\left|z_{j}\right|^{2} .
$$

This implies that $\lambda\left(\left[E_{j}^{*}, E_{j}\right]\right)<0$ for each $j=1,2, \ldots, m$.

Now, we give an explicit expression for the derived representation $d \pi_{\chi}$. If $L$ is a Lie group and $X$ is an element of the Lie algebra of $L$ then we denote by $X^{+}$the right invariant vector field on $L$ generated by $X$, that is, $X^{+}(h)=\left.\frac{d}{d t}(\exp t X) h\right|_{t=0}$ for $h \in L$.

By differentiating the multiplication map from $P^{+} \times K^{c} \times P^{-}$onto $P^{+} K^{c} P^{-}$, we can easily prove the following result.

Lemma 3.2. Let $X \in \mathrm{g}^{c}$ and $g=z k y$ where $z \in P^{+}, k \in K^{c}$ and $y \in P^{-}$. We have

(1) $d \zeta_{g}\left(X^{+}(g)\right)=\left(\operatorname{Ad}(z) p_{\mathfrak{p}^{+}}\left(\operatorname{Ad}\left(z^{-1}\right) X\right)\right)^{+}(z)$.

(2) $d \kappa_{g}\left(X^{+}(g)\right)=\left(p_{f^{c}}\left(\operatorname{Ad}\left(z^{-1}\right) X\right)\right)^{+}(k)$.

(3) $d \eta_{g}\left(X^{+}(g)\right)=\left(\operatorname{Ad}\left(k^{-1}\right) p_{\mathfrak{p}^{-}}\left(\operatorname{Ad}\left(z^{-1}\right) X\right)\right)^{+}(y)$.

From this, we deduce the following proposition (see also [36], p. 515).

Proposition 3.3. For $X \in \mathfrak{g}^{c}$ and $f \in \mathcal{H}_{\chi}$, we have $d \pi_{\chi}(X) f(Z)=d \chi\left(p_{\mathfrak{f}^{c}}\left(\operatorname{Ad}\left((\exp Z)^{-1}\right) X\right)\right) f(Z)-(d f)_{Z}\left(\frac{\operatorname{ad} Z}{1-e^{-\operatorname{ad} Z}} p_{\mathfrak{p}^{+}}\left(e^{-\operatorname{ad} Z} X\right)\right)$. 
In particular, if $\mathrm{g}$ is reductive then

(1) If $X \in \mathfrak{p}^{+}$then $d \pi_{\chi}(X) f(Z)=-(d f)_{Z}(X)$.

(2) If $X \in \mathfrak{f}^{c}$ then $d \pi_{\chi}(X) f(Z)=d \chi(X) f(Z)+(d f)_{Z}([Z, X])$.

(3) If $X \in \mathfrak{p}^{-}$then $d \pi_{\chi}(X) f(Z)=-d \chi([Z, X]) f(Z)-\frac{1}{2}(d f)_{Z}([Z,[Z, X]])$.

\section{Berezin symbols}

In this section, we introduce the Berezin calculus on $\mathcal{D}$ [8], [9] and we give explicit expressions for the Berezin symbols of $\pi_{\chi}(g), g \in G$ and of $d \pi_{\chi}(X), X \in \mathfrak{g}^{c}$ following the same lines as in [18].

Consider an operator (not necessarily bounded) $A$ on $\mathcal{H}_{\chi}$ whose domain contains $e_{Z}$ for each $Z \in \mathcal{D}$. Then the Berezin symbol of $A$ is the function $S_{\chi}(A)$ defined on $\mathcal{D}$ by

$$
S_{\chi}(A)(Z):=\frac{\left\langle A e_{Z}, e_{Z}\right\rangle_{\chi}}{\left\langle e_{Z}, e_{Z}\right\rangle_{\chi}}
$$

We can verify that an operator is determined by its Berezin symbol and that if an operator $A$ has adjoint $A^{*}$ then we have $S_{\chi}\left(A^{*}\right)=\overline{S_{\chi}(A)}$ [8], [23]. Moreover, we have the following equivariance property.

LEMMA 4.1. (1) For each $g \in G$ and each $Z \in \mathcal{D}$, we have $\pi_{\chi}(g) e_{Z}=$ ${\overline{J_{\chi}(g, Z)}}^{-1} e_{g \cdot Z}$.

(2) Let $A$ be an operator on $\mathcal{H}_{\chi}$ whose domain contains the coherent states $e_{Z}$ for each $Z \in \mathcal{D}$. Then, for each $g \in G$, the domain of $\pi_{\chi}\left(g^{-1}\right) A \pi_{\chi}(g)$ also contains $e_{Z}$ for each $Z \in \mathcal{D}$ and we have

$$
S_{\chi}\left(\pi_{\chi}(g)^{-1} A \pi_{\chi}(g)\right)(Z)=S_{\chi}(A)(g \cdot Z)
$$

for each $g \in G$ and $Z \in \mathcal{D}$.

Proof. (1) For $g \in G$ and $Z, W \in \mathcal{D}$, we have

$$
\begin{gathered}
\left\langle e_{W}, \pi_{\chi}(g) e_{Z}\right\rangle_{\chi}=\left\langle\pi_{\chi}\left(g^{-1}\right) e_{W}, e_{Z}\right\rangle_{\chi}=\left(\pi_{\chi}\left(g^{-1}\right) e_{W}\right)(Z) \\
=J_{\chi}(g, Z)^{-1} e_{W}(g \cdot Z)=J_{\chi}(g, Z)^{-1}\left\langle e_{W}, e_{g \cdot Z}\right\rangle_{\chi} .
\end{gathered}
$$

Then we have $\pi_{\chi}(g) e_{Z}={\overline{J_{\chi}(g, Z)}}^{-1} e_{g \cdot Z}$.

(2) This is an immediate consequence of (1). 
Proposition 4.2. (1) For $g \in G$ and $Z \in \mathcal{D}$, we have

$$
S_{\chi}\left(\pi_{\chi}(g)\right)(Z)=\chi\left(\kappa\left(\exp Z^{*} g^{-1} \exp Z\right)^{-1} \kappa\left(\exp Z^{*} \exp Z\right)\right) .
$$

(2) For $X \in \mathrm{g}^{c}$ and $Z \in \mathcal{D}$, we have

$$
S_{\chi}\left(d \pi_{\chi}(X)\right)(Z)=d \chi\left(p_{f^{c}}\left(\operatorname{Ad}\left(\zeta\left(\exp Z^{*} \exp Z\right)^{-1} \exp Z^{*}\right) X\right)\right) .
$$

In particular, if $\mathfrak{p}^{+}$is abelian and $X \in \mathfrak{f}^{c}$, we have

$$
S_{\chi}\left(d \pi_{\chi}(X)\right)(Z)=d \chi\left(X+p_{f^{c}}\left[\log \eta\left(\exp Z^{*} \exp Z\right),[X, Z]\right]\right) .
$$

Proof. (1) We have

$$
\begin{aligned}
& \left\langle\pi_{\chi}(g) e_{Z}, e_{Z}\right\rangle_{\chi}=\left(\pi_{\chi}(g) e_{Z}\right)(Z)=\overline{J(g, Z)}^{-1} e_{g \cdot Z}(Z) \\
& =c_{\chi} \overline{\chi(\kappa(g \exp Z))}^{-1} \chi\left(\kappa\left(\exp (g \cdot Z)^{*} \exp Z\right)\right)^{-1} .
\end{aligned}
$$

Now, we write

$$
g \exp Z=\exp (g \cdot Z) \kappa(g \exp Z) \eta(g \exp Z)
$$

Then we have

$$
\exp Z^{*} g^{*} \exp Z=\eta(g \exp Z)^{*} \kappa(g \exp Z)^{*} \exp (g \cdot Z)^{*} \exp Z .
$$

Thus, since $g^{*}=g^{-1}$, we get

$$
\kappa\left(\exp Z^{*} g^{-1} \exp Z\right)=\kappa(g \exp Z)^{*} \kappa\left(\exp (g \cdot Z)^{*} \exp Z\right)
$$

This gives

$$
\left\langle\pi_{\chi}(g) e_{Z}, e_{Z}\right\rangle_{\chi}=c_{\chi} \chi\left(\kappa\left(\exp Z^{*} g^{-1} \exp Z\right)\right)^{-1}
$$

hence the result.

(2) The result easily follows from the computation of the derivative of the function $S_{\chi}\left(\pi_{\chi}(\exp t X)\right)(Z)$ at $t=0$ by means of Lemma 3.2.

\section{Adapted Weyl correspondence}

In this section, we show that the Berezin calculus $A \rightarrow S_{\chi}(A)$ gives an adapted Weyl correspondence on the coadjoint orbit of $G$ associated with $\pi_{\chi}$. 
Recall that $\chi$ is a unitary character of $K$. Then the linear extension of $d \chi \in \mathfrak{f}^{*}$ to $\mathfrak{f}^{c}$ (also denoted by $d \chi$ ) is zero on $\mathfrak{g}_{\alpha}$ for each $\alpha \in \Delta_{c}$. Moreover we have $d \chi(\mathfrak{h}) \subset i \mathbb{R}$. Consider the linear form $\xi$ on $\mathrm{g}^{c}$ defined by $\xi=-i d \chi$ on $\mathfrak{f}^{c}$ and $\xi=0$ on $\mathfrak{p}^{ \pm}$. Then we have $\xi(\mathfrak{g}) \subset \mathbb{R}$ and the restriction $\xi_{0}$ of $\xi$ to $\mathfrak{g}$ is an element of $\mathrm{g}^{*}$. We denote by $\mathcal{O}\left(\xi_{0}\right)$ the orbit of $\xi_{0}$ in $\mathrm{g}^{*}$ for the coadjoint action of $G$. Then we have the following result.

Proposition 5.1.

(1) For each $X \in \mathrm{g}^{c}$ and each $Z \in \mathcal{D}$, we have

$$
S\left(d \pi_{\chi}(X)\right)(Z)=i\left\langle\psi_{\chi}(Z), X\right\rangle
$$

where $\psi_{\chi}(Z):=\operatorname{Ad}^{*}\left(\exp \left(-Z^{*}\right) \zeta\left(\exp Z^{*} \exp Z\right)\right) \xi_{0}$.

(2) For each $g \in G$ and each $Z \in \mathcal{D}$, we have $\psi(g \cdot Z)=\operatorname{Ad}^{*}(g) \psi(Z)$.

(3) The map $\psi$ is a diffeomorphism from $\mathcal{D}$ onto $\mathcal{O}\left(\xi_{0}\right)$.

Proof. Statement (1) is an immediate consequence of (2) of Proposition 4.2, and Statement (2) follows from Statement (1) and Lemma 4.1. In order to prove Statement (3), we first show that for $Z \in \mathcal{D}$, the equality $\psi_{\chi}(Z)=\xi_{0}$ implies $Z=0$.

Assume that $\psi_{\chi}(Z)=\xi_{0}$. Then we have $\operatorname{Ad}^{*}\left(\zeta\left(\exp Z^{*} \exp Z\right)\right) \xi_{0}=$ $\operatorname{Ad}^{*}\left(\exp Z^{*}\right) \xi_{0}$. This implies that

$$
\left\langle\xi_{0}, \operatorname{Ad}\left(\zeta\left(\exp Z^{*} \exp Z\right)^{-1}\right) Z\right\rangle=\left\langle\xi_{0}, \operatorname{Ad}\left(\exp \left(-Z^{*}\right)\right) Z\right\rangle .
$$

Thus, taking into account that $\left.\xi_{0}\right|_{\mathfrak{p}^{ \pm}}=0$, we find that $\left\langle\xi_{0},\left[Z^{*}, Z\right]\right\rangle=0$. Applying Lemma 3.1, we get $Z=0$.

Now, suppose that $\psi_{\chi}(Z)=\psi_{\chi}\left(Z^{\prime}\right)$ for some $Z, Z^{\prime} \in \mathcal{D}$. Choose $g, g^{\prime} \in G$ such that $g \cdot 0=Z$ and $g^{\prime} \cdot 0=Z^{\prime}$. By (2), we have $\operatorname{Ad}^{*}(g) \xi_{0}=\operatorname{Ad}^{*}\left(g^{\prime}\right) \xi_{0}$. Then we have $\operatorname{Ad}^{*}\left(g^{-1} g^{\prime}\right) \xi_{0}=\xi_{0}$ and by (2) again we get $\psi_{\chi}\left(\left(g^{-1} g^{\prime}\right) \cdot 0\right)=\xi_{0}$. By using the assertion already proved, we obtain that $\left(g^{-1} g^{\prime}\right) \cdot 0=0$. Thus we have $g^{-1} g^{\prime} \in K^{c} P^{-} \cap G=K$. Hence $Z=g \cdot 0=g^{\prime} \cdot 0=Z^{\prime}$. This proves that $\psi_{\chi}$ is injective. By using (2) we see that $\psi_{\chi}$ is also surjective hence bijective. It remains to show that $\psi_{\chi}$ is regular. Using (2) again, it is sufficient to verify that $\psi_{\chi}$ is regular at $Z=0$. But, using Lemma 3.2, we easily obtain that

$$
\left\langle\left(d \psi_{\chi}\right)_{0}(V), X\right\rangle=\left\langle\xi_{0},\left[X, V-V^{*}\right]\right\rangle
$$

for each $V \in \mathfrak{p}^{+}$and each $X \in \mathfrak{g}^{c}$. Assuming that $\left(d \psi_{\chi}\right)_{0}(V)=0$ for some $V \in \mathfrak{p}^{+}$and taking $X=V$ in the preceding equality, we get $\left\langle\xi_{0},\left[V, V^{*}\right]\right\rangle=0$ hence $V=0$ by Lemma 3.1 . 


\section{Stratonovich-Weyl correspondence}

We retain the notation from the previous sections. We denote by $\mathcal{L}_{2}\left(\mathcal{H}_{\chi}\right)$ the space of all Hilbert-Schmidt operators on $\mathcal{H}_{\chi}$. It is well-known that the map $S_{\chi}$ is a bounded operator from $\mathcal{L}_{2}\left(\mathcal{H}_{\chi}\right)$ into $L^{2}(\mathcal{D}, \mu)$ which is one-to-one and has dense range [39], [43].

The Berezin transform is the operator on $L^{2}(\mathcal{D})$ defined by $B_{\chi}:=S_{\chi} S_{\chi}^{*}$ or, equivalently, by the integral formula

$$
B_{\chi} F(Z)=\int_{\mathcal{D}} F(W) \frac{\left|\left\langle e_{Z}, e_{W}\right\rangle\right|_{\chi}^{2}}{\left\langle e_{Z}, e_{Z}\right\rangle_{\chi}\left\langle e_{W}, e_{W}\right\rangle_{\chi}} d \mu(W)
$$

(see [8], [43], [45] for instance). As a consequence of the equivariance property for $S_{\chi}, B_{\chi}$ commute with $\rho(g)$ for each $g \in G$, where $\rho$ is the leftregular representation of $G$ on $L^{2}\left(\mathcal{D}, \mu_{\chi}\right)$.

Now, we introduce the polar decomposition of $S_{\chi}: S_{\chi}=\left(S_{\chi} S_{\chi}^{*}\right)^{1 / 2} W_{\chi}=$ $B_{\chi}^{1 / 2} W_{\chi}$ where $W_{\chi}:=B_{\chi}^{-1 / 2} S_{\chi}$ is a unitary operator from $\mathcal{L}_{2}\left(\mathcal{H}_{\chi}\right)$ onto $L^{2}(\mathcal{D}, \mu)$. We immediately obtain the following proposition which is analogous to Theorem 3 of [27] and Proposition 3.1 of [20]. Note that, by (2) of Proposition 5.1, the measure $\mu_{0}:=\left(\psi_{\chi}^{-1}\right)^{*}(\mu)$ is a $G$-invariant measure on $\mathcal{O}\left(\xi_{0}\right)$.

Proposition 6.1. 1) The map $W_{\chi}: \mathcal{L}_{2}\left(\mathcal{H}_{\chi}\right) \rightarrow L^{2}\left(\mathcal{D}, \mu_{\chi}\right)$ is a Stratonovich-Weyl correspondence for the triple $\left(G, \pi_{\chi}, \mathcal{D}\right)$, that is, we have

(1) $W\left(A^{*}\right)=\overline{W(A)}$;

(2) $W\left(\pi(g) A \pi(g)^{-1}\right)(Z)=W(A)\left(g^{-1} \cdot Z\right)$;

(3) $W_{\chi}$ is unitary.

2) Similarly, the map $\mathcal{W}_{\chi}: \mathcal{L}_{2}\left(\mathcal{H}_{\chi}\right) \rightarrow L^{2}\left(\mathcal{O}\left(\xi_{0}\right), \mu_{0}\right)$ defined by $\mathcal{W}_{\chi}(A)=W_{\chi}(A) \circ \psi^{-1}$ is a Stratonovich-Weyl correspondence for the triple $\left(G, \pi_{\chi}, \mathcal{O}\left(\xi_{0}\right)\right)$.

Note that here we have relaxed the requirement that $W_{\chi}$ maps the identity operator $I$ to the constant function 1 (see Definition 1.2) since it is not adapted to the present setting where $I$ is not Hilbert-Schmidt. However, this requirement should be hold in some generalize sense, see [29].

In the rest of this section, we assume that $\mathfrak{g}$ is reductive. Then $\mathfrak{p}^{+}$and $\mathfrak{p}^{-}$are abelian, $\left[\mathfrak{p}^{+}, \mathfrak{p}^{-}\right] \subset \mathfrak{f}^{c}$ [36], p. 241 and $\mathcal{D}$ is bounded [36], p. 504. We will extend the Berezin transform to a class of functions which contains $S_{\chi}\left(d \pi_{\chi}(X)\right)$ for $X \in \mathrm{g}^{c}$ in order to define and study $W_{\chi}\left(d \pi_{\chi}(X)\right)$. 
We introduce some additional notation. Let $\left(E_{\alpha}\right)_{\alpha \in \Delta_{p}^{+}}$be a basis for $\mathfrak{p}^{+}$ such that $E_{\alpha} \in g_{\alpha}$ and $\alpha\left(\left[E_{\alpha}^{*}, E_{\alpha}\right]\right)=2$ for each $\alpha \in \Delta_{p}^{+}$. Let $\alpha_{1}, \alpha_{2}, \ldots, \alpha_{n}$ be an enumeration of $\Delta_{p}^{+}$. We write $Z=\sum_{k=1}^{n} z_{k} E_{\alpha_{k}}$ for the decomposition of $Z \in \mathfrak{p}^{+}$in the basis $\left(E_{\alpha_{k}}\right)$. If $f$ is a holomorphic function on $\mathcal{D}$, then we denote by $\partial_{k} f$ the partial derivative of $f$ with respect to $z_{k}$. We say that a function $f(Z)$ on $\mathcal{D}$ is a polynomial of degree $q$ in the variable $Z$ if $f\left(\sum_{k=1}^{n} z_{k} E_{\alpha_{k}}\right)$ is a polynomial of degree $q$ in the variables $z_{1}, z_{2}, \ldots, z_{n}$. For $Z, W \in \mathcal{D}$, we set $l_{Z}(W):=\log \eta\left(\exp Z^{*} \exp W\right) \in \mathfrak{p}^{-}$.

LEMMA 6.2 .

(1) For $Z, W \in \mathcal{D}$ and $V \in \mathfrak{p}^{+}$, we have

$$
\left.\frac{d}{d t} e_{Z}(W+t V)\right|_{t=0}=-e_{Z}(W) d \chi\left(\left[l_{Z}(W), V\right]\right) .
$$

and, for $Z, W \in \mathcal{D}$ and $V \in \mathfrak{p}^{+}$, we have

$$
\left.\frac{d}{d t} l_{Z}(W+t V)\right|_{t=0}=\frac{1}{2}\left[l_{Z}(W),\left[l_{Z}(W), V\right]\right] .
$$

(2) The function $\left(\partial_{k_{1}} \partial_{k_{2}} \cdots \partial_{k_{q}} e_{Z}\right)(W)$ is of the form $e_{Z}(W) P\left(l_{Z}(W)\right)$ where $P$ is a polynomial of degree $\leq q$.

(3) For each $X_{1}, X_{2}, \ldots, X_{q} \in \mathfrak{g}^{c}$, the operator $d \pi_{\chi}\left(X_{1} X_{2} \cdots X_{q}\right)$ is a sum of terms of the form $P(Z) \partial_{k_{1}} \partial_{k_{2}} \cdots \partial_{k_{q}}$ where $P$ is a polynomial in $Z$ of degree $\leq 2 q$.

(4) Each holomorphic differential operator on $\mathcal{D}$ with polynomial coefficients has Berezin symbol. In particular, for each $X_{1}, X_{2}, \ldots, X_{q} \in \mathrm{g}^{c}, S_{\chi}\left(d \pi_{\chi}\left(X_{1} X_{2} \cdots X_{q}\right)\right)$ is well-defined and is a sum of terms of the form $P(Z) Q\left(l_{Z}(Z)\right)$ where $P$ is a polynomial of degree $\leq 2 q$ and $Q$ is a polynomial of degree $\leq q$.

Proof. We verify (1) by a routine computation using Lemma 3.2. (2) is deduced from (1) by induction on $q$ and (3) follows from (2) and Proposition 3.3. Finally, (4) is a direct consequence of (2) and (3).

Let $\gamma_{1}, \gamma_{2}, \ldots, \gamma_{r}$ be a maximal orthogonal subset of $\Delta_{p}^{+}$as in [36], p. 503 . We set $q_{\chi}:=-\operatorname{Max}_{1 \leq s \leq r}\left(\lambda+\lambda_{0}\right)\left(\left[E_{\gamma_{r}}^{*}, E_{\gamma_{r}}\right]\right)$ where $\lambda_{0}=\left.d \chi_{0}\right|_{\mathfrak{h}}$. The following proposition is analogous to Proposition 4.1 of [20]. 
Proposition 6.3. If $q \leq q_{\chi}$ then for each $X_{1}, X_{2}, \ldots, X_{q} \in \mathfrak{g}^{c}$, the Berezin transform of $S_{\chi}\left(d \pi_{\chi}\left(X_{1} X_{2} \cdots X_{q}\right)\right)$ is well-defined.

Proof. We imitate the proof of Proposition 4.1 of [20]. We fix $Z \in \mathcal{D}$ and we choose $g \in G$ such that $g \cdot 0=Z$. By performing the change of variables $W \rightarrow g \cdot W$ in the integral (6.1) we get

$$
\begin{aligned}
\left(B_{\chi} F\right)(Z) & =\int_{\mathcal{D}} F(g \cdot W)\left\langle e_{W}, e_{W}\right\rangle_{\chi}^{-1} d \mu(W) \\
= & \int_{\mathcal{D}} F(g \cdot W) c_{\chi}\left(\chi \cdot \chi_{0}\right)\left(\kappa\left(\exp W^{*} \exp W\right)\right) d \mu_{L}(W)
\end{aligned}
$$

Note that if $F=S_{\chi}\left(d \pi_{\chi}\left(X_{1} X_{2} \cdots X_{q}\right)\right)$ then we have $F(g \cdot W)=$ $S_{\chi}\left(d \pi_{\chi}\left(Y_{1} Y_{2} \cdots Y_{q}\right)\right)(W)$ where $Y_{k}:=\operatorname{Ad}\left(g_{Z}^{-1}\right) X_{k}$ for $k=1,2, \ldots, q$. Now we verify that the condition $q \leq q_{\chi}$ implies that the function

$$
W \rightarrow S_{\chi}\left(d \pi_{\chi}\left(Y_{1} Y_{2} \cdots Y_{q}\right)\right)(W)\left(\chi \cdot \chi_{0}\right)\left(\kappa\left(\exp W^{*} \exp W\right)\right)
$$

is bounded hence integrable on the bounded domain $\mathcal{D}$. By Lemma 6.2, the function $S_{\chi}\left(d \pi_{\chi}\left(Y_{1} Y_{2} \cdots Y_{q}\right)\right)$ is a sum of terms of the form $P(W) Q\left(l_{W}(W)\right)$ where $P$ is a polynomial and $Q$ is a polynomial of degree $\leq q$. By [36], p. 504, each $W \in \mathcal{D}$ can be written as $W=\operatorname{Ad}(k)\left(\sum_{k=1}^{r} t_{s} E_{\gamma_{s}}\right)$ where $k \in K$ and $\left.t_{s} \in\right]-1,1\left[\right.$ for $s=1,2, \ldots r$. From the orthogonality of the roots $\gamma_{s}$, we get

$$
\left.\log \eta\left(\exp W^{*} \exp W\right)\right)=\operatorname{Ad}(k)\left(\sum_{s=1}^{r} \frac{t_{s}}{1-t_{s}^{2}} E_{\gamma_{s}}^{*}\right)
$$

and

$$
\kappa\left(\exp W^{*} \exp W\right)=k \exp \left(\sum_{s=1}^{r} \log \frac{1}{1-t_{s}^{2}}\left[E_{\gamma_{s}}^{*}, E_{\gamma_{s}}\right]\right) k^{-1} .
$$

Then we have

$$
\left(\chi \cdot \chi_{0}\right)\left(\kappa\left(\exp W^{*} \exp W\right)\right)=\prod_{s=1}^{r}\left(1-t_{s}^{2}\right)^{-\left(\lambda+\lambda_{0}\right)\left(\left[E_{\gamma_{s}}^{*}, E_{\gamma_{s}}\right]\right.} .
$$

Thus we see that the condition $q \leq q_{\chi}$ implies that the functions

$$
W \rightarrow P(W) Q\left(l_{W}(W)\right)\left(\chi \cdot \chi_{0}\right)\left(\kappa\left(\exp W^{*} \exp W\right)\right)
$$

are bounded hence the result. 
In particular, if $q_{\chi} \geq 1$ then the Berezin transform of $S_{\chi}\left(d \pi_{\chi}(X)\right)$ is welldefined for $X \in \mathrm{g}^{c}$. In the rest of this section, we study $B_{\chi} S_{\chi}\left(d \pi_{\chi}(X)\right)$ in order to define $W_{\chi}\left(d \pi_{\chi}(X)\right)$.

Since $\mathfrak{g}$ is reductive, we have the direct decomposition $\mathfrak{g}=\mathfrak{g}_{0} \oplus \mathfrak{g}_{1} \oplus \cdots \mathfrak{g}_{N}$ where $\mathfrak{g}_{0}=\mathcal{Z}(\mathfrak{g})$, the ideals $\mathfrak{g}_{1}, \ldots, \mathfrak{g}_{m}$ are non-compact and the ideals $\mathfrak{g}_{m+1}, \ldots, \mathfrak{g}_{N}$ are compact.

Let $j=m+1, \ldots, N$. Note that $\mathfrak{g}_{j} \subset \mathfrak{f}$. Then, since $\chi$ is a unitary character of $K$, we have $\lambda(X)=d \chi(X)=0$ for each $X \in \mathfrak{g}_{j}$. Moreover, for $X \in \mathfrak{g}_{j}$ and $Z \in \mathfrak{p}^{+}$, we have $[X, Z] \in \mathfrak{g}_{j} \cap \mathfrak{p}^{+} \subset \mathfrak{f}^{c} \cap \mathfrak{p}^{+}=(0)$. Therefore, by using Proposition 3.3 we see that $d \pi_{\chi}(X)=0$ for each $X \in \mathfrak{g}_{j}$.

The following proposition is the generalization of Proposition 5.2 in [20].

Proposition 6.4. The Berezin transform maps $S_{\chi}\left(d \pi_{\chi}\left(\mathrm{g}^{c}\right)\right)$ onto itself and for each $j=0,1, \ldots, m$ there exists a constant $c_{j}>0$ such that $B_{\chi} S_{\chi}\left(d \pi_{\chi}(X)\right)=c_{j} S_{\chi}\left(d \pi_{\chi}(X)\right)$ for each $X \in \mathfrak{g}_{j}^{c}$.

Proof. First we consider the linear form $b_{\chi}$ defined on $\mathrm{g}^{c}$ by

$$
b_{\chi}(X):=B_{\chi} S_{\chi}\left(d \pi_{\chi}(X)\right)(0)=\int_{\mathcal{D}} S_{\chi}\left(d \pi_{\chi}(X)\right)(Z) \chi\left(\kappa\left(\exp Z^{*} \exp Z\right)\right) d \mu(Z) .
$$

Changing variables $Z \rightarrow k^{-1} \cdot Z$ in this integral we see that $b_{\chi}(\operatorname{Ad}(k) X)=$ $b_{\chi}(X)$ for each $k \in K$ and each $X \in \mathfrak{g}^{c}$. In particular, for $X \in \mathfrak{g}_{\alpha}$ and $k=\exp H$ with $H \in \mathfrak{h}$ we get $e^{\alpha(H)} b_{\chi}(X)=b_{\chi}(X)$. Then we see that $b_{\alpha}(X)=0$ for each $X \in \mathfrak{g}_{\alpha}, \alpha \in \Delta$. Moreover, for each $H \in \mathfrak{h}^{c}$, we have $b_{\chi}(H)=c_{\chi}^{-1} \lambda(H)+\int_{\mathcal{D}} \lambda\left(\left[\log \eta\left(\exp Z^{*} \exp Z\right),[H, Z]\right]\right) \chi\left(\kappa\left(\exp Z^{*} \exp Z\right)\right) d \mu(Z)$.

Now, as in [36], p. 552, we consider the decomposition $\mathcal{D}_{1} \times \mathcal{D}_{2} \times \cdots \times \mathcal{D}_{m}$ corresponding to the direct decomposition $\mathfrak{g}=\mathfrak{g}_{0} \oplus \mathfrak{g}_{1} \oplus \cdots \mathfrak{g}_{N}$. For $j=1,2, \ldots, m$, we also set $\mathfrak{h}_{j}:=\mathfrak{h} \cap \mathfrak{g}_{j}, \lambda_{j}=\left.\lambda\right|_{\mathfrak{h}_{j}}$, etc. Then for each $H \in \mathfrak{h}_{j}$ we have

$b_{\chi}(H)=c_{\chi}^{-1} \lambda_{j}(H)+\int_{\mathcal{D}_{j}} \lambda_{j}\left(\left[\log \eta\left(\exp Z_{j}^{*} \exp Z_{j}\right),\left[H, Z_{j}\right]\right]\right) \chi_{j}\left(\kappa\left(\exp Z_{j}^{*} \exp Z_{j}\right)\right) d \mu_{j}\left(Z_{j}\right)$,

with obvious notation. Therefore, by [20], Proposition 5.1, there exists a constant $c_{j}>0$ such that $b_{\chi}(H)=c_{j} \lambda_{j}(H)$ for each $H \in \mathfrak{h}_{j}$. This implies that $B_{\chi}\left(S_{\chi}\left(d \pi_{\chi}(X)\right)\right)(0)=c_{j} S_{\chi}\left(d \pi_{\chi}(X)\right)(0)$ for each $X \in \mathfrak{g}_{j}$. Finally, replacing $X$ by $\operatorname{Ad}\left(g^{-1}\right) X$ and applying equivariance (see Lemma 4.1) we obtain the desired result. 
As a consequence of this proposition, we can define $B_{\chi}^{-1 / 2}$ on the space of functions $\left\{S_{\chi}\left(d \pi_{\gamma}(X)\right): X \in \mathfrak{g}^{c}\right\}$ and then $W_{\chi}$ on the space $\left\{d \pi_{\chi}(X): X \in \mathrm{g}^{c}\right\}$. More precisely, for each $j=0,1, \ldots, m$ we have $W_{\chi}\left(d \pi_{\chi}(X)\right)=c_{j}^{-1 / 2} S_{\chi}\left(d \pi_{\chi}(X)\right)$ for each $X \in \mathrm{g}_{j}^{c}$. Note that $c_{0}=1$ but the explicit computation of the constants $c_{j}$ for $j>0$ is not easy in general, see Section 6 of [20] for the case $G=S U(p, q)$.

\section{Example: the diamond group}

In this section we show by considering the case of the diamond group that, in general, one cannot expect to get results similar to those obtained in Section 6 for g reductive.

We fix an integer $n \geq 1$. The diamond (real) Lie algebra $g$ is the semidirect product of $\mathbb{R}$ with the $(2 n+1)$-dimensional Heisenberg Lie algebra. More precisely, $g$ has basis $\left\{H, \tilde{Z}, X_{1}, \ldots, X_{n}, Y_{1}, \ldots, Y_{n}\right\}$, the non-trivial brackets being given by

$$
\left[H, X_{k}\right]=-Y_{k} ; \quad\left[H, Y_{k}\right]=X_{k} ; \quad\left[X_{k}, Y_{k}\right]=\tilde{Z} .
$$

Let $G$ be the connected and simply connected (real) Lie group with Lie algebra g. Then $G$ is a non-exponential solvable Lie group. Each $g \in G$ can be written uniquely as

$$
g=\exp t H \exp \left(\sum_{k} x_{k} X_{k}+\sum_{k} y_{k} Y_{k}+s \tilde{Z}\right)
$$

where $t, s, x_{k}$ and $y_{k}$ are real numbers. Then we denote $g=\left(t, s,\left(x_{k}\right),\left(y_{k}\right)\right)$. The group law of $G$ is given by

$$
\left(t, s,\left(x_{k}\right),\left(y_{k}\right)\right) \cdot\left(t^{\prime}, s^{\prime},\left(x_{k}^{\prime}\right),\left(y_{k}^{\prime}\right)\right)=\left(t^{\prime \prime}, s^{\prime \prime},\left(x_{k}^{\prime \prime}\right),\left(y_{k}^{\prime \prime}\right)\right)
$$

where

$$
\begin{aligned}
& t^{\prime \prime}=t+t^{\prime} \\
& s^{\prime \prime}=s+s^{\prime}+\frac{1}{2} \sum_{k}\left(\cos t^{\prime}\left(x_{k} y_{k}^{\prime}-x_{k}^{\prime} y_{k}\right)-\sin t^{\prime}\left(x_{k} x_{k}^{\prime}+y_{k} y_{k}^{\prime}\right)\right) \\
& x_{k}^{\prime \prime}=x_{k}^{\prime}+x_{k} \cos t^{\prime}-y_{k} \sin t^{\prime} \\
& y_{k}^{\prime \prime}=y_{k}^{\prime}+x_{k} \sin t^{\prime}+y_{k} \cos t^{\prime} .
\end{aligned}
$$

Let $\left\{H^{*}, \tilde{Z}^{*}, X_{1}^{*}, \ldots, X_{n}^{*}, Y_{1}^{*}, \ldots, Y_{n}^{*}\right\}$ be the dual basis of $\mathrm{g}^{*}$. The coadjoint action of $g=\left(t, s,\left(x_{k}\right),\left(y_{k}\right)\right) \in G$ on $\xi=\left[d, c,\left(a_{k}\right),\left(b_{k}\right)\right]:=d H^{*}+c \tilde{Z}^{*}+$ 
$\sum_{k} a_{k} X_{k}^{*}+\sum_{k} b_{k} Y^{*}$ is given by $\operatorname{Ad}^{*}(g) \xi=\left[d^{\prime}, c^{\prime},\left(a_{k}^{\prime}\right),\left(b_{k}^{\prime}\right)\right]$ where $c^{\prime}=c$ and

$$
\begin{aligned}
& d^{\prime}=d+\frac{c}{2} \sum_{k}\left(x_{k}^{2}+y_{k}^{2}\right)+\sum_{k}\left(a_{k} y_{k}-b_{k} x_{k}\right) \\
& a_{k}^{\prime}=a_{k} \cos t+b_{k} \sin t-c\left(x_{k} \sin t-y_{k} \cos t\right) \\
& b_{k}^{\prime}=-a_{k} \sin t+b_{k} \cos t-c\left(x_{k} \cos t+y_{k} \sin t\right) .
\end{aligned}
$$

From this, it follows that if a coadjoint orbit of $G$ contains a point of the form $\left[d, 0,\left(a_{k}\right),\left(b_{k}\right)\right]$ then it is trivial or diffeomorphic to $\mathbb{R} \times \mathbb{S}^{1}$. Moreover, if a coadjoint orbit contains a point $\left[d, c,\left(a_{k}\right),\left(b_{k}\right)\right]$ with $c \neq 0$ then it has dimension $2 n$ and contains a unique point of the form $[d, c,(0),(0)]$ with $c \neq 0$. Such an orbit is called generic.

In the rest of this section, we fix $\xi_{0}=\left[d_{0}, c_{0},(0),(0)\right] \in \mathfrak{g}^{*}$ such that $c_{0} \neq 0$ and we consider the generic coadjoint orbit $\mathcal{O}\left(\xi_{0}\right)$ of $\xi_{0}$. We assume that $c_{0}>0$ (the case where $c_{0}<0$ can be treated similarly).

The subalgebra $\mathfrak{h}=\operatorname{span}\{H, \tilde{Z}\}$ is a compactly embedded Cartan subalgebra of $\mathfrak{g}$. Here we have $\mathfrak{f}=\mathfrak{h}$. We choose the positive root to be $\alpha$ defined by $\alpha(H)=-i$. Then we see that $\mathfrak{p}^{+}$has basis $\left(E_{j}\right)_{1 \leq j \leq n}$ where $E_{j}=1 / 2\left(X_{j}-i Y_{j}\right)$. We write $Z=\sum_{j} z_{j} E_{j}$ for the decomposition of $Z \in \mathfrak{p}^{+}$
in this basis.

The $P^{+} K^{c} P^{-}$-decomposition of $g=\left(t, s,\left(x_{k}\right),\left(y_{k}\right)\right) \in G$ is given by

$$
g=\exp \sum_{k} a_{k}\left(X_{k}-i Y_{k}\right) \exp (u H+v \tilde{Z}) \exp \sum_{k} b_{k}\left(X_{k}+i Y_{k}\right)
$$

where $\quad u=t, \quad v=s-(i / 4) \sum_{k}\left(x_{k}^{2}+y_{k}^{2}\right), \quad a_{k}=1 / 2\left(x_{k}+i y_{k}\right) e^{-i t} \quad$ and $b_{k}=1 / 2\left(x_{k}-i y_{k}\right)$.

From this, we deduce that if $Z=\sum_{j} z_{j} E_{j}$ then we have

$$
\kappa\left(\exp Z^{*} \exp Z\right)=\exp \left(\frac{i}{2}|Z|^{2} \tilde{Z}\right)
$$

where $|Z|=\left(\sum_{j}\left|z_{j}\right|^{2}\right)^{1 / 2}$. Moreover the action of $\left(t, s,\left(x_{k}\right),\left(y_{k}\right)\right) \in G$ on $Z=\sum_{j} z_{j} E_{j} \in \mathcal{D}$ is given by $g \cdot Z=\sum_{j}\left(z_{j}+x_{j}+i y_{j}\right) e^{-i t} E_{j}$. Note that here we have $\mathcal{D}=\mathfrak{p}^{+}$.

Since we have $\left[H, E_{j}\right]=-i E_{j}$ for each $j=1,2, \ldots, n$, we immediately see that $\operatorname{Tr}_{\mathfrak{p}^{+}} \operatorname{ad}(u H+v \tilde{Z})=-i n u$ for each $u, v \in \mathbb{C}$. The character $\chi_{0}$ is then $\chi_{0}(\exp (u H+v \tilde{Z}))=e^{-i n u}$. In particular we have $\chi_{0}\left(\kappa\left(\exp Z^{*} \exp Z\right)\right)=1$ for each $Z \in \mathfrak{p}^{+}$. The $G$-invariant measure on $\mathcal{D}=\mathfrak{p}^{+}$is then the Lebesgue measure $\mu_{L}$. Here we normalize $\mu_{L}$ as follows. We take $d \mu_{L}(Z)=$ $(2 \pi)^{-n} c_{0}^{n} d x_{1} d y_{1} \cdots d x_{n} d y_{n}$ where $Z=\sum_{j}\left(x_{j}+i y_{j}\right) E_{j}, x_{j}, y_{j} \in \mathbb{R}$. 
We consider the character $\chi$ of $K$ defined by $\chi(\exp (u H+v \tilde{Z}))=$ $e^{i\left(d_{0} u+c_{0} v\right)}$ for $u, v \in \mathbb{R}$. Then we have $\left.d \chi\right|_{\mathfrak{f}}=\left.i \xi_{0}\right|_{\mathfrak{f}}$. We can easily verify that

$$
e_{Z}(W)=\chi^{-1}\left(\kappa\left(\exp Z^{*} \exp W\right)\right)=e^{\frac{c_{0}}{2}(W, Z\rangle}
$$

with the notation $\langle W, Z\rangle:=\sum_{j} \bar{z}_{j} w_{j}$ for $Z=\sum_{j} z_{j} E_{j}$ and $W=\sum_{j} w_{j} E_{j}$ in $\mathfrak{p}^{+}$.
We also have

$$
J(g, Z)=\exp i\left(d_{0} t+c_{0} s\right) \exp \left(\frac{c_{0}}{2} \sum_{k} z_{k}\left(x_{k}-i y_{k}\right)+\frac{c_{0}}{4} \sum_{k}\left(x_{k}^{2}+y_{k}^{2}\right)\right)
$$

for $Z \in \mathfrak{p}^{+}$and $g=\left(t, s,\left(x_{k}\right),\left(y_{k}\right)\right) \in G$. Note that we have $c_{\chi}=1$ here. Therefore, $\pi_{\chi}$ is given by

$$
\begin{array}{r}
\pi_{\chi}(g) f(Z)=\exp i\left(d_{0} t+c_{0} s\right) \exp \left(-\frac{c_{0}}{4} \sum_{k}\left(x_{k}^{2}+y_{k}^{2}\right)\right) \exp \left(\frac{c_{0}}{2} \sum_{k} z_{k}\left(x_{k}-i y_{k}\right) e^{i t}\right) \\
\times f\left(\sum_{k}\left(z_{k} e^{i t}-x_{k}-i y_{k}\right) E_{k}\right)
\end{array}
$$

for $Z \in \mathfrak{p}^{+}, g=\left(t, s,\left(x_{k}\right),\left(y_{k}\right)\right) \in G$ and $f \in \mathcal{H}_{\chi}$. Note that the norm of $\mathcal{H}_{\chi}$ is defined by

$$
\|f\|_{\chi}^{2}=\int_{\mathfrak{p}^{+}}|f(Z)|^{2} e^{-\frac{c_{0}}{2}|Z|^{2}} d \mu_{L}(Z) .
$$

We can verify that $\pi_{\chi}$ can be also obtained from $\mathcal{O}\left(\xi_{0}\right)$ by using the general method of [5], taking the positive polarization $\operatorname{span}_{\mathrm{C}}\left\{H, \tilde{Z}, X_{j}+i Y_{j}, 1 \leq j \leq n\right\}$ at $\xi_{0}$, see [7], p. 189-190.

By differentiating $\pi_{\chi}$ we get

$$
\begin{aligned}
& S_{\chi}\left(d \pi_{\chi}(H)\right)(Z)=i d_{0}+\frac{1}{2} i c_{0}|Z|^{2} \\
& S_{\chi}\left(d \pi_{\chi}(\tilde{Z})\right)(Z)=i c_{0} \\
& S_{\chi}\left(d \pi_{\chi}\left(X_{k}\right)\right)(Z)=\frac{1}{2} c_{0}\left(z_{k}-\overline{z_{k}}\right) \\
& S_{\chi}\left(d \pi_{\chi}\left(Y_{k}\right)\right)(Z)=-\frac{i}{2} c_{0}\left(z_{k}+\overline{z_{k}}\right) .
\end{aligned}
$$

This gives

$$
\psi(Z)=\left(d_{0}+\frac{1}{2} c_{0}|Z|^{2}\right) H^{*}+c_{0} \tilde{Z}^{*}+\sum_{k} c_{0} \frac{z_{k}-\overline{z_{k}}}{2 i} X_{k}^{*}-\sum_{k} c_{0} \frac{z_{k}+\overline{z_{k}}}{2} Y_{k}^{*} .
$$


Note that $\psi(Z)=\operatorname{Ad}^{*}(g) \xi_{0}$ where $g=\left(t, 0, \operatorname{Re}\left(e^{i t} Z\right), \operatorname{Im}\left(e^{i t} Z\right)\right)$. Note also that the Berezin symbol of $\pi_{\chi}(g)$ is

$$
\begin{aligned}
S_{\chi}\left(\pi_{\chi}(g)\right)(Z) & =\exp i\left(d_{0} t+c_{0} s\right) \exp \left(-\frac{c_{0}}{2}|Z|^{2}\right) \exp \left(-\frac{c_{0}}{4} \sum_{k}\left(x_{k}^{2}+y_{k}^{2}\right)\right) \\
& \times \exp \left(\frac{c_{0}}{2} e^{i t} \sum_{k} z_{k}\left(x_{k}-i y_{k}\right)\right) \exp \left(\frac{c_{0}}{2} e^{i t}|Z|^{2}\right) \\
& \times \exp \left(-\frac{c_{0}}{2} \sum_{k}\left(x_{k}+i y_{k}\right) \overline{z_{k}}\right)
\end{aligned}
$$

for $g=\left(t, s,\left(x_{k}\right),\left(y_{k}\right)\right) \in G$ and $Z \in \mathfrak{p}^{+}$.

The Berezin transform is given by

$$
\begin{aligned}
B_{\chi}(F)(Z) & =\int_{\mathfrak{p}^{+}} e^{\frac{c_{0}}{2}\left(\langle W, Z\rangle+\langle Z, W\rangle-|Z|^{2}-|W|^{2}\right)} F(W) d \mu_{L}(W) \\
& =\int_{\mathfrak{p}^{+}} e^{-\frac{c_{0}}{2}|W|^{2}} F(Z-W) d \mu_{L}(W) .
\end{aligned}
$$

In particular, we consider the function $F_{\gamma, U}$ defined by $F_{\gamma, U}(Z)=$ $e^{i \gamma \operatorname{Re}\langle Z, U\rangle}$ for $\gamma \in \mathbb{C}$ and $U \in \mathfrak{p}^{+}$such that $|U|=1$. Then we have

$$
B_{\chi}\left(F_{\gamma, U}\right)(Z)=F_{\gamma, U}(Z) \int_{\mathfrak{p}^{+}} e^{-\frac{c_{0}}{2}}|W|^{2} e^{-i \gamma \operatorname{Re}\langle W, W\rangle} d \mu_{L}(W)=e^{-\gamma^{2} / 2 c_{0}} F_{\gamma, U}(Z) .
$$

On the other hand, let us introduce the Laplacian $L:=4 \sum_{k=1}^{n} \frac{\partial^{2}}{\partial z_{k} \partial \bar{z}_{k}}$. Clearly $L\left(F_{\gamma, U}\right)=-\gamma^{2} F_{\gamma, U}$. Then $B_{\chi}\left(F_{\gamma, U}\right)=\exp \left(L / 2 c_{0}\right)\left(F_{\gamma, U}\right)$ for each $\gamma, U$ and we have recovered the well-known formula $B_{\chi}=\exp \left(L / 2 c_{0}\right)$, see the introduction of [43] for instance. We are now in position to compute $W_{\chi}\left(d \pi_{\chi}(X)\right)$ for $X \in \mathfrak{g}$. Indeed, one has

$$
W_{\chi}\left(d \pi_{\chi}(X)\right)=B_{\chi}^{-1 / 2} S_{\chi}\left(d \pi_{\chi}(X)\right)=\exp \left(-L / 4 c_{0}\right) S_{\chi}\left(d \pi_{\chi}(X)\right) .
$$

Then we immediately obtain $W_{\chi}\left(d \pi_{\chi}(X)\right)=S_{\chi}\left(d \pi_{\chi}(X)\right)$ for $X=X_{1}, \ldots, X_{n}$, $Y_{1}, \ldots, Y_{n}, \tilde{Z}$ and

$$
W_{\chi}\left(d \pi_{\chi}(H)\right)=S_{\chi}\left(d \pi_{\chi}(H)\right)-\frac{1}{4 c_{0}} L\left(S_{\chi}\left(d \pi_{\chi}(H)\right)\right)=S_{\chi}\left(d \pi_{\chi}(H)\right)-\frac{1}{2} i n .
$$

In particular, we cannot find a basis $\left(X_{j}\right)$ of $\mathfrak{g}$ for which there exists a family $\left(c_{j}\right)$ of real numbers such that $W_{\chi}\left(d \pi_{\chi}\left(X_{j}\right)\right)=c_{j} S_{\chi}\left(d \pi_{\chi}\left(X_{j}\right)\right)$ for each $j$, as this is the case when $g$ is reductive, see Section 6 . 


\section{REFERENCES}

[1] S. T. Ali - M. EngLis, Quantization methods: a guide for physicists and analysts, Rev. Math. Phys. 17, 4 (2005), pp. 391-490.

[2] J. Arazy - H. Upmeier, Weyl Calculus for Complex and Real Symmetric Domains, Harmonic analysis on complex homogeneous domains and Lie groups (Rome, 2001). Atti Accad. Naz. Lincei Cl. Sci. Fis. Mat. Natur. Rend. Lincei (9) Mat. Appl. 13, no 3-4 (2002), pp. 165-181.

[3] J. ARAZY - H. UPMEIER, Invariant symbolic calculi and eigenvalues of invariant operators on symmeric domains, Function spaces, interpolation theory and related topics (Lund, 2000), pp. 151-211, de Gruyter, Berlin, 2002.

[4] D. ARnal - J.-C. Cortet, Nilpotent Fourier Transform and Applications, Lett. Math. Phys. 9 (1985), pp. 25-34.

[5] L. Auslander - B. Kostant, Polarization and Unitary Representations of Solvable lie Groups, Invent. Math. 14 (1971), pp. 255-354.

[6] I. Beltiță - D. Beltiță, Magnetic pseudo-differential Weyl calculus on nilpotent Lie groups, Ann. Global Anal. Geom. 36, 3 (2009), pp. 293-322.

[7] P. Bernat - N. Conze - M. Duflo - M. Levy-Nahas - M. Rais - P. Renouard M. Vergne, Representations des groupes de Lie résolubles, Dunod, Paris 1972.

[8] F. A. BerezIN, Quantization, Math. USSR Izv. 8, 5 (1974), pp. 1109-1165.

[9] F. A. BEREZIN, Quantization in complex symmetric domains, Math. USSR Izv. 9, 2 (1975), pp. 341-379.

[10] C. BRIF - A. MANN, Phase-space formulation of quantum mechanics and quantum-state reconstruction for physical systems with Lie-group symmetries, Phys. Rev. A 59, 2 (1999), pp. 971-987.

[11] B. CAHEn, Deformation Program for Principal Series Representations, Lett. Math. Phys. 36 (1996), pp. 65-75.

[12] B. CAHEN, Quantification d'une orbite massive d'un groupe de Poincaré généralisé, C. R. Acad. Sci. Paris t. 325, série I (1997), pp. 803-806.

[13] B. Cahen, Weyl quantization for semidirect products, Differential Geom. Appl. 25 (2007), pp. 177-190.

[14] B. CAHEN, Weyl quantization for principal series, Beiträge Algebra Geom. 48, 1 (2007), pp. 175-190.

[15] B. CaHen, Contraction of compact semisimple Lie groups via Berezin quantization, Illinois J. Math. 53, 1 (2009), pp. 265-288.

[16] B. CAHEN, Berezin quantization on generalized flag manifolds, Math. Scand. 105 (2009), pp. 66-84.

[17] B. CAHEN, Contraction of discrete series via Berezin quantization, J. Lie Theory, 19 (2009), pp. 291-310.

[18] B. CAHEN, Berezin quantization for discrete series, Beiträge Algebra Geom. 51 (2010), pp. 301-311.

[19] B. CAHEN, Stratonovich-Weyl correspondence for compact semisimple Lie groups, Rend. Circ. Mat. Palermo, 59 (2010), pp. 331-354.

[20] B. CAHEN, Stratonovich-Weyl correspondence for discrete series representations, Arch. Math. (Brno), 47 (2011), pp. 41-58.

[21] B. CAHEN, Weyl quantization for the semi-direct product of a compact Lie group and a vector space, Comment. Math. Univ. Carolin. 50, 3 (2009), pp. $325-347$. 
[22] B. Cahen, Weyl quantization for Cartan motion groups, Comment. Math. Univ. Carolin. 52, 1 (2011), pp. 127-137.

[23] M. CAHEN - S. GUTT - J. RAWnsley, Quantization on Kähler manifolds I, Geometric interpretation of Berezin quantization, J. Geom. Phys. 7 (1990), pp. 45-62.

[24] J. F. CARIÑena - J. M. GRacia-Bondìa - J. C. VÀrilly, Relativistic quantum kinematics in the Moyal representation, J. Phys. A: Math. Gen. 23 (1990), pp. 901-933.

[25] P. Cotton - A. H. Dooley, Contraction of an Adapted Functional Calculus, J. Lie Theory, 7 (1997), pp. 147-164.

[26] M. Davidson - G. Olafsson - G. Zhang, Laplace and Segal-Bargmann transforms on Hermitian symmetric spaces and orthogonal polynomials, J. Funct. Anal. 204 (2003), pp. 157-195.

[27] H. FigueroA - J. M. Gracia-BondìA - J. C. VÀrilly, Moyal quantization with compact symmetry groups and noncommutative analysis, J. Math. Phys. 31 (1990), pp. 2664-2671.

[28] B. Folland, Harmonic Analysis in Phase Space, Princeton Univ. Press, 1989.

[29] J. M. GRACIA-BondìA, Generalized Moyal quantization on homogeneous symplectic spaces, Deformation theory and quantum groups with applications to mathematical physics (Amherst, MA, 1990), pp. 93-114, Contemp. Math., 134, Amer. Math. Soc., Providence, RI, 1992.

[30] J. M. Gracia-Bondìa - J. C. VÀrilly, The Moyal Representation for Spin, Ann. Physics, 190 (1989), pp. 107-148.

[31] M. GotaY, Obstructions to Quantization, in: Mechanics: From Theory to Computation (Essays in Honor of Juan-Carlos Simo), J. Nonlinear Science Editors, Springer New-York, 2000, pp. 271-316.

[32] S. Helgason, Differential Geometry, Lie Groups and Symmetric Spaces, Graduate Studies in Mathematics, Vol. 34, American Mathematical Society, Providence, Rhode Island 2001.

[33] A. W. KnapP, Representation theory of semi-simple groups. An overview based on examples, Princeton Math. Series t. 36 (1986).

[34] A. A. KirILlov, Lectures on the Orbit Method, Graduate Studies in Mathematics Vol. 64, American Mathematical Society, Providence, Rhode Island, 2004.

[35] B. Kostant, Quantization and unitary representations, in: Modern Analysis and Applications, Lecture Notes in Mathematics 170, Springer-Verlag, Berlin, Heidelberg, New-York, 1970, pp. 87-207.

[36] K-H. NeEB, Holomorphy and Convexity in Lie Theory, de Gruyter Expositions in Mathematics, Vol. 28, Walter de Gruyter, Berlin, New-York 2000.

[37] T. Nomura, Berezin Transforms and Group representations, J. Lie Theory, 8 (1998), pp. 433-440.

[38] B. ØRSTED - G. ZHANG, Weyl Quantization and Tensor Products of Fock and Bergman Spaces, Indiana Univ. Math. J. 43, 2 (1994), pp. 551-583.

[39] J. Peetre - G. Zhang, A weighted Plancherel formula III. The case of a hyperbolic matrix ball, Collect. Math. 43 (1992), pp. 273-301.

[40] N. V. Pedersen, Matrix coefficients and a Weyl correspondence for nilpotent Lie groups, Invent. Math. 118 (1994), pp. 1-36. 
[41] I. SATAKE, Algebraic structures of symmetric domains, Iwanami Sho-ten, Tokyo and Princeton Univ. Press, Princeton, NJ, 1971.

[42] R. L. Stratonovich, On distributions in representation space, Soviet Physics. JETP, 4 (1957), pp. 891-898.

[43] A. Unterberger - H. Upmeier, Berezin transform and invariant differential operators, Commun. Math. Phys. 164, 3 (1994), pp. 563-597.

[44] N. J. WildBERGER, Convexity and unitary representations of a nilpotent Lie group, Invent. Math. 89 (1989), pp. 281-292.

[45] G. ZHANG, Berezin transform on compact Hermitian symmetric spaces, Manuscripta Math. 97 (1998), pp. 371-388.

Manoscritto pervenuto in redazione il 19 Marzo 2012. 
